\title{
El Grupo de Trabajo Interdisciplinar Patrimonio Cartográfico en las IDE
}

Joan Capdevila Subirana, Instituto Geográfico Nacional

\section{INTRODUCCIÓN}

Las cosas que pasan, pasan en algún lugar. Esta simple y obvia afirmación explica en gran manera el espectacular éxito que está teniendo la divulgación de cartografía a través de la red. Y no tan sólo en lo concerniente a mapas e imágenes del territorio. En este apartado también debemos considerar toda aquella información vinculada a una posición sobre la superficie terrestre, lo que llamamos información geográfica.

Este éxito no hubiera sido posible sin el desarrollo de las TIG ni sin su aprovechamiento por las grandes empresas especializadas en difundir información a través de Internet, como es el caso de Google con Google Maps y Google Earth, Microsoft con Bing! y otras. Sin embargo, presentan un gran defecto de fondo: son ofertas privadas y privativas, que responden a razones estrictamente comerciales.

Ello explica el porqué la Administración Pública y una buena parte de la industria del sector de la información geográfica está apostando por una estrategia diferente: las Infraestructuras de Datos Espaciales (IDE). Bajo esta denominación se quiere designar un nuevo paradigma para la publicación de datos geográficos en la red. Este engloba varios elementos: un marco político proactivo, que estimule la reutilización de la información; un marco organizativo que busque la armonización de datos entre productores y la generación de metadatos; y un marco técnico que promueva el desarrollo de tecnologías consensuadas e independientes de sistemas comerciales. Todo el sistema es vertebrado por un concepto básico, verdadero motor de las IDE: la interoperabilidad. Es decir, la capacidad de intercambio de información entre sistemas informáticos con los mínimos condicionantes técnicos posibles.

Todos y cada uno de estos elementos se vienen desarrollando desde los años noventa, aunque su aplicación sólo es patente desde principios del presente siglo (CROMPVOETS; BREGT, 2006). No entraremos en detalles, simplemente mencionaremos el apoyo que están teniendo a todos los niveles de la administración: la comunidad europea ha publicado directivas estimulando su creación, España ha legislado recientemente en este sentido y las comunidades autónomas también lo están haciendo (RODRÍGUEZ, 2010). Los proyectos de armonización de datos están en todas la agendas de las instituciones responsables. Continuamente están apareciendo nuevos geoportales ${ }^{1}$ y se están mejorando los existentes. $Y$ todo con un único fin: publicar información geográfica en la red de la forma más transparente y útil para el usuario.

\section{PROBLEMAS ENTRE CARTOGRAFÍA HISTÓRICA E IDE}

Nadie puede dudar que la cartografia histórica es un dato geográfico y, por ello, susceptible de ser publicado en Internet utilizando los mecanismos preconizados por las IDE. Sin embargo, esta publicación no se está dando². Podemos achacarlo a varias razones. En primer lugar, no existe cobertura legal que estimule la publicación de cartografía histórica mediante las IDE. El apoyo político es esencial para garantizar los recursos necesarios para mantener en marcha una IDE pero la cartografía histórica aún no tiene un reflejo en la legislación mencionada. Por otro lado, hay que reconocer que los principales desarrollos en IDE están claramente enfocados a satisfacer la creciente demanda de información geográfica actualizada. Ello también sería un elemento que justificaria el escaso tratamiento de lo temporal que se percibe en las IDE y, en general, en el mundo de la información geográfica. Tanto en las aplicaciones como en los servicios se puede afirmar que el tiempo es un atributo de datos y metadatos poco cuidado. Y, finalmente, no debemos olvidar la distancia metodológica y técnica existente entre las comunidades de profesionales dedicados a la preservación de documentación y aquellos vinculados al desarrollo de las IDE. Vienen de tradiciones y ámbitos de conocimiento muy diferentes.

\section{EL GRUPO DE TRABAJO INTERDISCIPLINAR PATRIMONIO CARTOGRÁFICO EN LAS IDE}

El desarrollo de las IDE en España está siendo impulsado por el Consejo Superior Geográfico ${ }^{3}$ desde 2002 a través de la Comisión Especializada en IDE. En el seno de esta Comisión se plantearon las cuestiones citadas y se convino en promover la incorporación de bibliotecas, cartotecas y archivos al ámbito de las IDE. Durante 2007 y 2008 se mantuvieron reuniones con diversos actores para perfilar líneas de actuación y se llegó a la conclusión de que la mejor manera de enfrentar el problema era la creación de un grupo de trabajo interdisciplinar, en el que participasen personas de ambas comunidades profesionales. La finalidad del grupo sería 


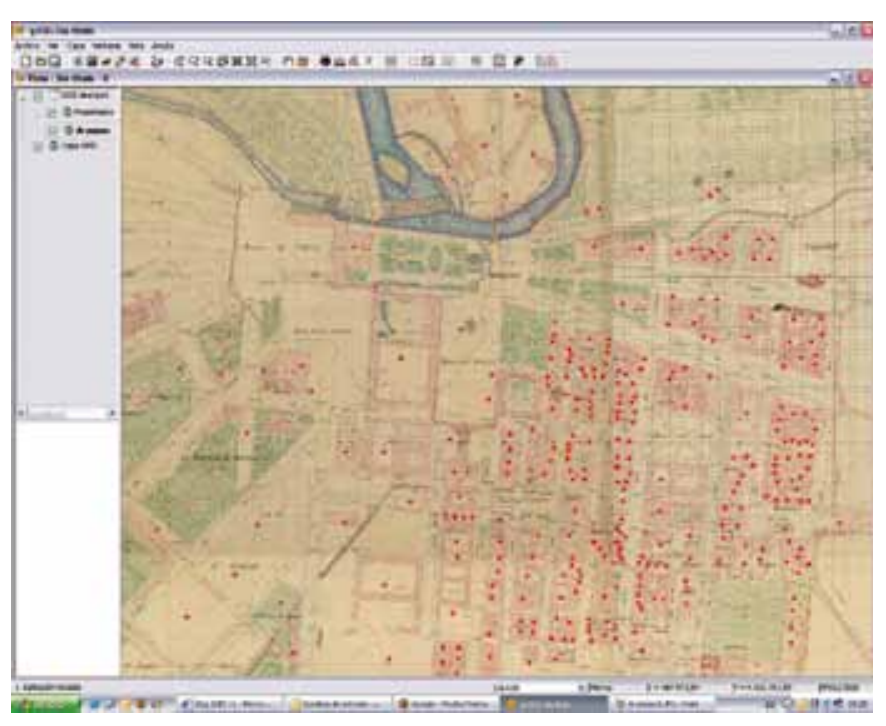

Ejemplo de visualización de cartografía histórica mediante un servicio web al que se ha accedido a través de un Sistema de Información Geográfica

la de definir y desarrollar proyectos compartidos para facilitar la publicación de cartografía histórica mediante las IDE.

En junio de 2008 se constituyó el Grupo de Trabajo Interdisciplinar Patrimonio Cartográfico en las IDE (GTI PC-IDE). En ese mismo año se presentó la iniciativa en IBERCARTO4. Con las aportaciones de ambas comunidades, a partir de noviembre de 2008 se pudo considerar GTI PC-IDE plenamente formado.

El primer reto fue el de intentar definir el objeto sobre el que trabaja el grupo, el patrimonio cartográfico. Tras algunos debates, se consensuó la siguiente definición:

"En el marco del GTI PC-IDE, entendemos por Patrimonio Cartográfico todos aquellos documentos que contienen información geográfica, que ya no cumplen con el propósito para los que fueron creados y que son susceptibles de reutilización", donde se entiende por información geográfica toda aquella "información relacionada tanto implícita como explícitamente con fenómenos asociados a una localización en la Tierra" ${ }^{5}$, donde el término propósito tiene el sentido considerado en el documento ISO $19115^{6}$ y el término reutilización tiene el sentido considerado en la Ley 37/2007, de 16 de noviembre, sobre reutilización de la información del sector público.

\section{LA PASARELA MARC21 - NEM/INSPIRE}

El primer proyecto planteado fue el de conseguir la interoperabilidad entre la familia de formatos de catalogación MARC y el formato de metadatos definidos por la norma ISO 19115. Para conseguirlo, se consideró necesario desarrollar una pasarela informática entre ambos. De hecho, tanto MARC como ISO 19115 son muy genéricos y, en la práctica, se suele trabajar con perfiles adaptados a las posibilidades y necesidades de los usuarios. El grupo tomó la decisión de concentrar sus esfuerzos en el paso de MARC21 (perfil de MARC) al Núcleo Español de Metadatos (conocido como NEM, un perfil de ISO 19115), que posteriormente se vería complementado con la norma de ejecución de metadatos derivada de la Directiva INSPIRE?.

Ya existian algunos antecedentes: Chandler, Foley y Hafez (2000) desarrollaron una pasarela entre MARC21 y Dublin Core, otro perfil de ISO 19115. Sin embargo, este caso no cubria los aspectos de georeferenciación que son propios de la cartografía histórica. Aprovechando el congreso Digital Technologies in Cartographic Heritage $^{8}$, celebrado en Barcelona en junio de 2008, se organizó una mesa redonda donde se respaldó la creación de la pasarela (MONTANER, 2009).

Se decidió llevar a cabo, por lo tanto, un desarrollo partiendo de cero. Para ello se utilizó el esquema conceptual descrito por uno de los miembros del Grupo (NOGUERAS-ISO; ZARAZAGA-SORIA; BÉJAR et ál., 2004) que descompone el trabajo en cuatro pasos: armonización entre formatos, definición de relaciones cruzadas o mapeo, establecimiento de reglas de conversión e implementación informática.

El primer paso para poder establecer una relación entre ambos formatos fue el de describirlos de forma similar. La armonización consistió en codificar los estándares mediante un esquema común, en sendas tablas, de manera que fueran comparables.

El siguiente paso consistió en el establecimiento de las relaciones cruzadas entre ambas tablas, proceso conocido también como mapeo. En ese momento aparecieron las primeras dificultades serias: era necesario saber exactamente cuál era el significado y el uso que se daba a cada campo en cada tabla. Tal como se constató, no todas las instituciones utilizaban MARC21 de la misma manera.

Para superar esta dificultad se contó con la participación de la empresa DMSGroup, especializada en catalogación mediante metadatos IDE. Conociendo el formato NEM/INSPIRE tuvieron que dilucidar como establecer las relaciones con MARC21. Para ello, se pidió a las cartotecas y archivos del grupo que llevaran a cabo la catalogación de diversos mapas históricos. También se llevaron a cabo varias encuestas entre los miembros del GTI PC-IDE planteándoles los problemas detectados y pidiéndoles su opinión sobre la mejor manera de abordarlos. Las soluciones pasaron por enriquecer las listas controladas manejadas por ambos estándares, introducir nuevas palabras clave y plantear el uso de tesauros para que la introducción tanto de las listas controladas como de palabras clave no modifiquen los estándares vigentes (CRESPO; CRIAD0; CAPDEVILA et ál., 2010).

En conclusión, se obtuvo un cruce total de 64 elementos pertenecientes a MARC21 frente a 60 elementos pertenecientes a ISO 19115. El 65\% de los elementos de NEM se puede obtener de 
MARC21. En el caso del perfil de metadatos de INSPIRE, este dato sube al $73 \%$.

El tercer paso consistió en establecer una serie de reglas de conversión imprescindibles para materializar informáticamente los cruces planteados. Cada regla intenta documentar y solucionar cada uno de los muchos casos que se pueden dar: el cruce de un elemento en un formato con varios del otro formato; la existencia de duplicidades innecesarias; el uso adecuado de las listas de palabras controladas o del tesauro; la automatización de rellenar los campos "no disponibles"; el tratamiento adecuado de las fechas; el tratamiento y el cruce de abreviaturas, etcétera.

Hasta aqui el desarrollo teórico de la pasarela, que fue presentado simultáneamente en la reunión de IBERCARTO 2010 (28 y 29 de octubre, Valencia) y en las Jornadas Ibéricas de IDE 2010 (27, 28 y 29 de octubre, Lisboa). Se trata de una primera versión. Cuenta con el consenso necesario dentro del GTI PC-IDE como para que se pueda plantear su materialización informática y se pueda poner a prueba con registros reales.

\section{PRÓXIMOS TRABAJOS}

El cuarto paso consiste en la puesta en marcha de la pasarela. Para ello se han planteado dos líneas de trabajo. Por un lado, el desarrollo de una implementación de referencia. Por otro, llevar a cabo un caso de uso.

La implementación de referencia es necesaria para acompañar la recomendación de la pasarela. Se considera una buena práctica el que cualquier propuesta informática pueda ir acompañada de una aplicación que permita comprobar su bondad. En este caso, se optará por un desarrollo basado en software libre, lo que va a permitir que la codificación de la pasarela esté disponible para todos aquellos interesados en integrarla en sus sistemas.

El caso de uso va a permitir comprobar la robustez de la pasarela. Consistirá en implementarla dentro de una institución que contenga un catálogo en MARC21. Esperamos ver en la práctica cuáles son los datos que se pierden en el proceso de transformación y cuáles son los problemas con que se pueden encontrar los usuarios directos, los documentalistas y catalogadores. La experiencia servirá, además, de ejemplo para otras instituciones para entender tanto las ventajas como las dificultades en el uso de la pasarela. Esperamos poder aprovechar estas enseñanzas para mejorar tanto la pasarela como su implementación informática.

Finalmente, es de esperar que la pasarela acabe siendo adoptada por el Consejo Superior Geográfico como recomendación y sirva de punto de arranque para que el patrimonio cartográfico se incorpore al desarrollo de las IDE. Tal como se ha visto, se trata de un elemento esencial para la publicación de cartografía histórica en la red mediante servicios interoperables. Pero no es suficiente, por lo que desde el GTI PC-IDE aún nos queda un largo camino para poner al alcance de todo el mundo, sobre el mundo, el valioso tesoro cartográfico custodiado en nuestros archivos.

\section{Notas}

${ }^{1}$ A modo de ejemplo, mencionar el geoportal de la IDE de España (www.idee.es) y el de la IDE de Andalucia (www.ideandalucia.es/).

2 Los ejemplos son escasos. Véase CAPDEVILA; BONILLA, 2009 o el n. ${ }^{\circ}$ 40, correspondiente a 2010, de la Revista Catalana de Geografia (www.rcg.cat).

${ }^{3}$ Consejo Superior Geográfico: www.fomento.es/MFOM/LANG_CASTELLANO/ ORGANOS_COLEGIADOS/CSG/

${ }^{4}$ IBERCARTO: www.sge.org/cartografia-ibercarto/ibercarto.html

${ }^{5}$ Definición tomada del glosario del ISO/TC 211.

${ }^{6}$ Diccionario de datos, apéndice B.2.1, de la norma ISO 19115:2003 sobre metadatos para la información geográfica.

${ }^{7}$ REGLAMENTO 1205/2008 de la Comisión, de 3 de diciembre de 2008, por el que se ejecuta la Directiva 2007/2/CE del Parlamento Europeo y del Consejo en lo que se refiere a los metadatos.

${ }^{8}$ Organizado por una comisión de la International Cartographic Association (xeee. web.auth.gr/ICA-Heritage/Commission/3rd_Workshop/Barcelona.htm).

\section{Bibliografía}

CAPDEVILA, J.; BONILLA, R. (2009) Cartographic patrimony in the Spanish SDI. The cadastral series of nineteenth century: Hojas kilométricas (Kilometric Sheets). e-Perimetron [en linea], 2009, v. 4, n. ${ }^{0}$ 1, pp. 38-44 <http://www.e-perimetron. org/> [05/11/2010]

CHANDLER, A.; FOLEY, D.; HAFEZ, A. M. (2000) Mapping and Converting Essential Federal Geographic Data Committee (FGDC) Metadata into MARC21 and Dublin Core. D-Lib Magazine [en línea], Enero 2000, vol. 6, n. $1<$ http://www.dlib. org $>[05 / 11 / 2010]$

CRESPO, M.; CRIADO, M.; CAPDEVILA, J. et ál. (2010) El Patrimonio Cartográfico en las Infraestructuras de Datos Espaciales Pasarela de metadatos MARC21 - ISO 19115 [comunicación]. En Jornadas Ibéricas de Infraestructuras de Datos, Lisboa, 27-29 de octubre, 2010

CROMPVOETS, J.; BREGT, A. (2006) Worldwide Development of National Spatial Data Clearinghouses (2000-2005) [comunicación]. En GSDI-9 Conference Proceedings, 6-10 November 2006, Santiago, Chile

MONTANER, C. (2009) Disseminating Digital Cartographic Heritage: Standards and infrastructures. e-Perimetron [en línea], 2009, v. 4, n. ${ }^{\circ}$, pp. 53-54 $<$ http://www.e-perimetron.org/> [05/11/2010] NOGUERAS-ISO, J.; ZARAZAGA-SORIA, F. J.; LACASTA, J. et ál. (2004). Metadata standard interoperability: application in the geographic information domain.Computers, Environment and Urban Systems [en línea], 2004, v. 28, n. ${ }^{\circ}$, pp. 611-634

RODRíguEZ, J. I. (2010) Precisa, útil y accesible a todos. Revista del Ministerio de Fomento, n. 598,2010 , pp. 30-37 